\title{
Cloridrato de benzocaína na anestesia de carpas (Cyprinus carpio)
}

\section{Benzocaine hydrochloride anesthesia in carp (Cyprinus carpio)}

\author{
Mariana Isa Poci Palumbo Antunes ${ }^{1}$; Rafael Sanches Spurio²; Daniella Aparecida \\ Godoi $^{2}$; Carmen Esther Santos Grumadas ${ }^{3}$; Marco Antonio da Rocha ${ }^{4 *}$
}

\section{Resumo}

\begin{abstract}
A anestesia em peixes é indicada para permitir a realização de diversos procedimentos como: biometria, marcação, transporte, exame físico, procedimentos cirúrgicos e manejo reprodutivo. Determinou-se a dose de benzocaína na anestesia de carpas (Cyprinus carpio), com a realização de seis etapas com 40 peixes cada. O peso médio das carpas em cada etapa foi de 147,45 $\pm 7,99 \mathrm{~g}, 173,32 \pm 9,15 \mathrm{~g}, 191,26 \pm 14,05 \mathrm{~g}$, $269,84 \pm 19,24 \mathrm{~g}, 285,25 \pm 17,97 \mathrm{~g}$, e $300,91 \pm 16,45 \mathrm{~g}$. Em cada etapa, os peixes foram capturados e colocados em quatro recipientes com benzocaína nas concentrações de 100,140,180 e $220 \mathrm{mg} / \mathrm{L}$ respectivamente. O tempo de indução (TI) foi registrado para cada peixe e após a indução anestésica foi realizada a biometria. Em cada etapa foi calculada a dose mínima de benzocaína através do Linear Response Plateau (LRP), em um modelo que incluiu dose e TI. O LRP foi calculado para cada fase, e os valores encontrados foram: $125,79 \mathrm{mg} / \mathrm{L}$ em 114,33s, $155,68 \mathrm{mg} / \mathrm{L} \mathrm{em} \mathrm{115,75s,} 145,33 \mathrm{mg} / \mathrm{L} \mathrm{em} \mathrm{102,52s,} 149,50 \mathrm{mg} / \mathrm{L} \mathrm{em} \mathrm{140,53s,}$ $166,42 \mathrm{mg} / \mathrm{L}$ em 116,15s e 158,34mg/L em 102,00s. A dose ótima foi relacionada com o peso, resultando na equação: dose $=114,230+0,158 \times$ peso $\left(r^{2}=0,53\right)$. A equação mostra que um aumento no peso em $1 \mathrm{~g}$, corresponde a um aumento de $0,158 \mathrm{mg} / \mathrm{L}$ na dose de cloridrato de benzocaína para carpas.
\end{abstract}

Palavras-chave: Peixes, carpa, benzocaína, anestesia

\begin{abstract}
Fish anesthesia is indicated to allow the accomplishment of several procedures such as biometry, tagging, transportation, physical examination, surgical procedures, and reproductive management. The doses of benzocaine in the carp anesthesia (Cyprinus carpio) were determined, carrying through six phases with 40 fish each. The average weight of carps in each phase was of $147.45 \pm 7.99 \mathrm{~g}, 173.32 \pm 9.15 \mathrm{~g}, 191.26 \pm 14.05 \mathrm{~g}$, $269.84 \pm 19.24 \mathrm{~g}, 285.25 \pm 17.97 \mathrm{~g}$, and $300.91 \pm 16.45 \mathrm{~g}$. In each phase, fish had been captured and placed in four containers each one with different concentrations of benzocaine (100,140, 180 and $220 \mathrm{mg} / \mathrm{L}$ respectively). The induction time (IT) was registered for each fish and after that the anesthetic induction biometry was performed. In each phase the minimal dose of benzocaine was calculated using the Linear Response Plateau (LRP), in a model that included dose and IT. The LRP was calculated for each phase: $125.79 \mathrm{mg} / \mathrm{L}$ in $114.33 \mathrm{~s}, 155.68 \mathrm{mg} / \mathrm{L}$ in $115.75 \mathrm{~s}, 145.33 \mathrm{mg} / \mathrm{L}$ in $102.52 \mathrm{~s}, 149.50 \mathrm{mg} / \mathrm{L}$ in $140.53 \mathrm{~s}, 166.42 \mathrm{mg} / \mathrm{L}$ in $116.15 \mathrm{~s}$, and $158.34 \mathrm{mg} / \mathrm{L}$ in $102.00 \mathrm{~s}$. The optimal dose was related with the weight, resulting in the equation: dose $=114.230+0.158 \mathrm{x}$ weight $\left(\mathrm{r}^{2}=0.53\right)$. The equation shows that an increase in the weight in $1 \mathrm{~g}$ corresponds to an increase of $0.158 \mathrm{mg} / \mathrm{L}$ in the dose of benzocaine hydrochloride for carps.
\end{abstract}

Key words: Fish, carp, benzocaine, anesthesia

1 Acadêmica de graduação em Medicina Veterinária da UEL, Londrina, PR.

2 Acadêmicos de graduação em Medicina Veterinária da UEL, Londrina, PR.

3 Professora Doutora, Departamento de Clínicas Veterinárias da UEL, Londrina, PR.

4 Professor Doutor, Departamento de Zootecnia da UEL, Londrina, PR - marco@uel.br.

* Autor para correspondência

Recebido para publicação 15/07/07 Aprovado em 16/1 1/07 


\section{Introdução}

A Piscicultura é uma atividade em grande expansão nacional e as técnicas de cultivo devem ser desenvolvidas para as nossas condições ambientais. Diversos agentes químicos são usados para a realização de anestesia em peixes. As doses recomendadas variam conforme o agente utilizado, a via de administração, a espécie do peixe e a qualidade da água (temperatura, concentração de oxigênio e constituição iônica). Sabe-se que a resistência e a tolerância aos anestésicos variam de indivíduo para indivíduo e que mesmo espécies afins podem diferir muito a esse respeito (HIKASA et al., 1986). Os procedimentos anestésicos podem ser utilizados visando à realização da biometria de peixes, marcação, corte de nadadeiras, transporte, exame físico e procedimentos cirúrgicos (HUBBELL; MUIR; SKARDA, 1989).

A anestesia geral em peixes foi descrita inicialmente por Mc Farland em 1960 (apud BROWN, 1988). Em 1969, Mc Farland e Klontz (apud THURMON; TRANQUILLI; BENSON, 1996) fizeram uma nova classificação da anestesia geral em peixes descrevendo-a em seis estágios: no estágio 1 (sedação leve), ocorre discreta perda da reação a estímulos externos visuais e táteis e equilíbrio normal; no estágio 2 (sedação profunda), ocorre perda total da reação a estímulos externos exceto pressão intensa, aparece uma pequena diminuição na freqüência opercular e o equilíbrio é normal; no estágio 3 (perda parcial do equilíbrio), há diminuição parcial do tono muscular, nado desincronizado, aumento da freqüência opercular, e reação somente a estímulos táteis e vibratórios intensos; no estágio 4 (perda total do equilíbrio) verifica-se perda total do tono muscular e equilíbrio, freqüência opercular rápida (ou lenta com alguns agentes anestésicos) e reação somente a estímulos de pressão intensos; no estágio 5 (perda da atividade reflexa), ocorre perda total da atividade reflexa, os movimentos operculares tornam-se muito superficiais e a freqüência cardíaca encontra-se baixa; no estágio 6 (colapso medular), os movimentos operculares cessam após um período de dificuldade respiratória, seguido por parada cardíaca.

A benzocaína (éster etílico do ácido paraaminobenzóico) é um anestésico local utilizado em mamíferos, e em peixes é usado como anestésico geral por imersão (MEINERTZ, 1999). A benzocaína é insolúvel em água (HEAVNER, 1996). Segundo a University of Michigan (2005), ela pode ser diluída em etanol ou acetona e estocada para posterior utilização na indução anestésica, dissolvida em água.

Tem sido indicada a adição de solução de benzocaína com etanol ou acetona à água na dose de 25-100 mg/L, dependendo da anestesia requerida. Os peixes podem ser manuseados quando perdem o equilíbrio e flutuam com a parte ventral do corpo para cima. Ao término do manuseio, os animais recuperados são imersos em água saturada de oxigênio. Os parâmetros farmacocinéticos da benzocaína aumentam com a elevação da temperatura da água (MEINERTZ, 1999).

A benzocaína tem sido utilizada para tranqüilização, analgesia e anestesia de peixes de várias espécies, tendo sido observada margem de segurança muito pequena entre a dose eficaz e a dose letal (OLFERT; CROSS; MCWILLIAM, 1993). A indução da anestesia com benzocaína ocorre entre dois a quatro minutos, e a recuperação em tempo inferior a dez minutos. Há a indicação das doses para diferentes espécies de peixes: 25 a 45 $\mathrm{mg} / \mathrm{L}$ para salmão; 55 a $80 \mathrm{mg} / \mathrm{L}$ para striped bass; 50 a $100 \mathrm{mg} / \mathrm{L}$ para carpa e $40 \mathrm{mg} / \mathrm{L}$ para bacalhau (OLFERT; CROSS; MCWILLIAM, 1993).

Brown (1988) utilizou a benzocaína na dose de 15 a $30 \mathrm{mg} / \mathrm{L}$ para sedação, e 30 a $50 \mathrm{mg} / \mathrm{L}$ para a manutenção de anestesia cirúrgica de peixes. Ferreira, Schoonbee e Smit (1984) indicam o uso de benzocaína na dose de 50 a $100 \mathrm{mg} / \mathrm{L}$ para a anestesia de peixes. Conforme Hubbell, Muir e Skarda (1989), a benzocaína pode ser utilizada como agente anestésico de peixes na dose de 25 a $100 \mathrm{mg} / \mathrm{L}$ de água.

Como geralmente existe uma diferença pequena entre a dose anestésica e a letal da benzocaína, 
pretende-se diminuir as taxas de mortalidade com o uso da mesma, conhecendo-se a dose desta para carpas. A determinação da dose anestésica de benzocaína para carpas criadas em tanques na Universidade Estadual de Londrina poderá ser utilizada como um padrão, facilitando o manejo desses peixes.

\section{Material e Métodos}

O experimento foi realizado na Estação de Piscicultura da Universidade Estadual de Londrina. Foram utilizadas 240 carpas (Cyprinus carpio), divididas em seis etapas com 40 peixes cada. $\mathrm{O}$ intervalo entre cada etapa foi de dois meses para garantir a ausência de anestésico residual. Os peixes de mesma desova foram retirados do tanque externo com superfície de $100 \mathrm{~m}^{2}$ e profundidade de $1,2 \mathrm{~m}$, e colocados em tanques menores, com capacidade para 1000 litros de água. Depois eram capturados ao acaso e colocados individualmente em bacias de plástico contendo água e o anestésico nas concentrações a serem testadas.

A temperatura da água foi mantida em torno de $23^{\circ} \mathrm{C}$. Em cada etapa foram realizados quatro tratamentos: 500, 700, 900 e 1100 mg de benzocaína diluída em álcool e depositada em bacias contendo 5 $\mathrm{L}$ de água. O tempo de indução (TI), definido como o tempo em segundos entre a colocação do peixe no tanque e o desaparecimento dos movimentos operculares, foi registrado para cada peixe. Após a indução anestésica, cada peixe foi mantido fora da água por 10 minutos, para realização da biometria. $\mathrm{O}$ peso corporal foi determinado em gramas.

Os peixes foram colocados em um tanque com renovação contínua de água para permitir a sua recuperação. Em cada etapa foi determinada a dose mínima de benzocaína através do Linear Response Plateau (LRP), usando o aplicativo SAEG (Sistema para Análise Estatística e Genética), em um modelo que incluiu dose e TI como variáveis. Ao final do experimento foi determinada a relação entre a dose de benzocaína $(\mathrm{mg} / \mathrm{L})$ pelo LRP e o peso corporal (gramas) das carpas através de regressão linear.

\section{Resultados e Discussão}

O peso médio dos peixes, o valor do LRP calculado para cada etapa e os respectivos tempos de indução (TI) são apresentados na Tabela 1. A média dos tempos de indução anestésica observados nesse estudo foi de 115,21 segundos; esse valor está ligeiramente abaixo do intervalo de tempo (120 a 240 segundos) indicado por Olfert, Cross e McWilliam(1993), e dentro dos limites citados pela University of Michigan (2005) para anestesia, cirurgia, analgesia e cuidados pósanestésicos em peixes (60 a 180 segundos).

Tabela 1. Peso corporal médio, dose mínima de benzocaína e tempo de indução encontrados em cada etapa do projeto, com a utilização do LRP.

\begin{tabular}{ccc}
\hline Peso (g) & Dose mínima de Benzocaína $\mathbf{( m g / L )}$ & Tempo de indução (s) \\
\hline 147,45 & 125,79 & 114,33 \\
173,32 & 155,68 & 115,75 \\
191,26 & 145,33 & 102,52 \\
269,84 & 149,50 & 140,53 \\
285,25 & 166,42 & 116,15 \\
300,91 & 158,34 & 102,00 \\
\hline
\end{tabular}

O presente trabalho relacionou a dose ótima com o peso, obtendo a equação: dose $=114,230+0,158 \times$ peso $\left(r^{2}=0.53\right)$ (Gráfico 1). Esse resultado indica que um aumento no peso em $1 \mathrm{~g}$, corresponde a um aumento de $0,158 \mathrm{mg} / \mathrm{L}$ na dose anestésica de cloridrato de benzocaína para carpas. A dose anestésica de benzocaína aqui determinada poderá ser utilizada visando diminuir os índices de mortalidade dos peixes com o uso da mesma. 


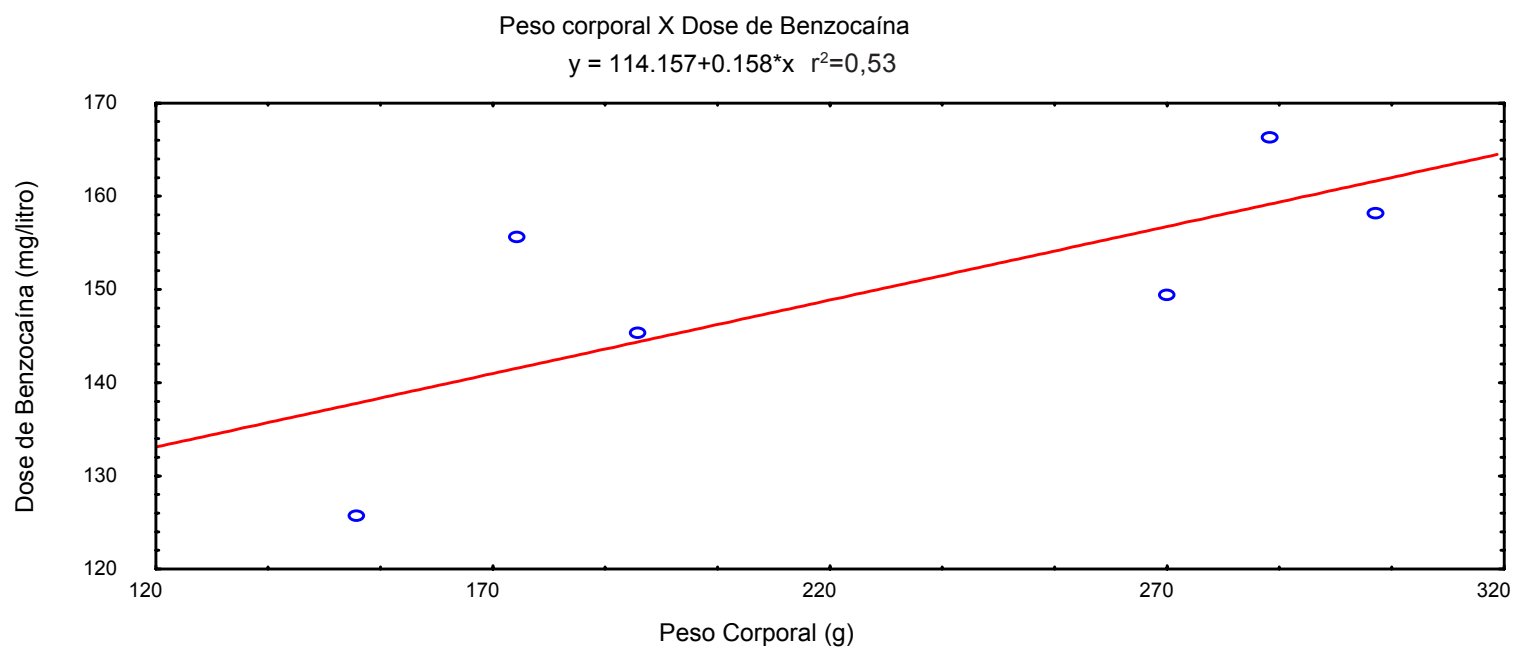

Gráfico 1. Relação entre a dose mínima de benzocaína e o peso corporal das carpas.

A anestesia de peixes tem sido indicada para imobilizá-los para realização de diversos procedimentos e verificou-se na literatura que as doses de benzocaína indicadas para carpas são muito variáveis. As doses testadas no presente estudo foram de $100,140,180$ e $220 \mathrm{mg} / \mathrm{L}$, e todas se mostraram seguras e eficazes.

O ‘Office Animal Resources Anesthetics'(2005) e 'Drug Dosages for Fish'(2005) indicam a utilização da dose de $25-50 \mathrm{mg} / \mathrm{L}$ de benzocaína para anestesiar peixes. Já no Veterinary Formulary (2005) da Universidade de Minnesota há a indicação de 100$200 \mathrm{mg} / \mathrm{L}$ de benzocaína, dose que está de acordo com o presente resultado.

Segundo os autores Olfert Cross e McWillian (1993) tem sido observada uma dose de segurança muito pequena entre a dose eficaz e a dose letal de Cloridrato de Benzocaína. Como no presente estudo não ocorreu óbito de nenhum peixe, podemos afirmar que as concentrações utilizadas de 100, 140, $180 \mathrm{e}$ $220 \mathrm{mg} / \mathrm{L}$ são seguras para induzir a anestesia em carpas.

O Manual de Procedimentos Operacionais publicado pelo 'Australian Aquatic Animal Disease Emergency Plan' apud Barker, (1999) recomenda a adição de benzocaína à água para produzir anestesia profunda, seguida de morte em peixes. O plano australiano de destruição de animais aquáticos indica que após a dissolução da benzocaína em etanol (1g/ 10L), a solução obtida deve ser diluída em água (1:1000) e misturada no tanque antes da anestesia, atingindo a concentração final de $100 \mathrm{mg} / \mathrm{L}$, que é suficiente para matar os peixes. Os métodos utilizados para a eutanásia de peixes na 'University of Michigan'(2005) incluem a imersão, por dez minutos ou mais em tanques contendo benzocaína em concentrações maiores que $100 \mathrm{mg} / \mathrm{L}$ de água.

\section{Conclusões}

Os resultados permitem concluir que partindo de uma dose mínima de $125,79 \mathrm{mg} / \mathrm{ml}$ e peso de $147,45 \mathrm{~g}$, um aumento no peso da carpa em $1 \mathrm{~g}$, corresponde a um aumento de $0,158 \mathrm{mg} / \mathrm{L}$ na dose de cloridrato de benzocaína para se obter a anestesia em Cyprinus carpio.

\section{Referências}

BROWN, L. A. Anesthesia in fish. Veterinary Clinics of North America: Small Animal Practice, Philadelphia, v.18, n.2, p.317-329, 1988.

DRUG DOSAGES FOR FISH. Office of animal research services. Disponível em: <http://www.oars.uconn.edu $>$. Acesso em: 6 dez. 2005. 
FERREIRA, J. T.; SCHOONBEE, H. J.; SMIT, G. L. The uptake of the anaesthetic benzocaine hydrochloride by the gills and the skin of three freshwater fish species. Journal of Fish Biology, London, v.25, n.1, p.35-41, jul. 1984.

HEAVNER, J. E. Local anesthetics. In: THURMON, J. C.; TRANQUILLI, W. J.; BENSON, G. J. Lumb\&Jones' veterinary anesthesia. 3.ed. Baltmore: Williams \& Wilkins, 1996. p.330-336.

HIKASA, Y.; TAKASE, K.; OGASAWARA, T.; OGASAWARA, S. Anesthesia and recovery with tricaine methanesulfonate, eugenol and thiopental sodium in the carp. Cyprinus Carpio. Nippon Juigaku Zasshi, Towada, v.48, n.2, p.341-351, 1986.

HUBBELL, J. A. E.; MUIR, W. W.; SKARDA, R. Anesthetic procedures and techniques in birds, fish, reptiles, amphibians, rodents and exotic cats. In: MUIR, W. W.; HUBBELL, J. A. E. Handbook of veterinary anesthesia. St Louis: Mosby, 1989. p.234-259.

MEINERTZ, I. R. Metabolism, elimination, and pharmacokinetics of the fish anesthetic benzocaine. In: SMITH, D. J.; GINGERICH, W. H.; BECONI-BARKER, M. G. Xenobiotics in fish. Dordrecht: Klumer Academic Publishers, 1999. p.189-200.
OFFICE ANIMAL RESOURCES ANESTHETICS: anesthesia for various animals. Disponível em: $<\mathrm{http}: /$ www.vetmed.auburn.edu>. Acesso em: 6 dez. 2005.

OLFERT, E. D.; CROSS, B. M.; MCWILliAM, A. A. Anesthetic and sedative drug dosage- fishes. In:

Guide to the care and use of experimental animals. 2.ed. Ottawa: Canadian Council on Animal Care, 1993. p.192.

THURMON, J. C.; TRANQUILLI, W. J.; BENSON, G. J. Anesthesia of wild, exotic, and laboratory animals. In: . Lumb\&Jones veterinary anesthesia. 3.ed. Baltmore: Williams \& Wilkins, 1996. p.686-735.

UNIVERSITY OF MICHIGAN. Method of euthanasia by species. Disponível em: <http://www.iacuc.anzona.edu/ training/surgery/euthanasia.html $>$. Acesso em: 6 dez. 2005.

VETERINARY Formulary. Disponível em:<http:// www.ahc.umn.edu/rar>. Acesso em: 6 dez. 2005. 
